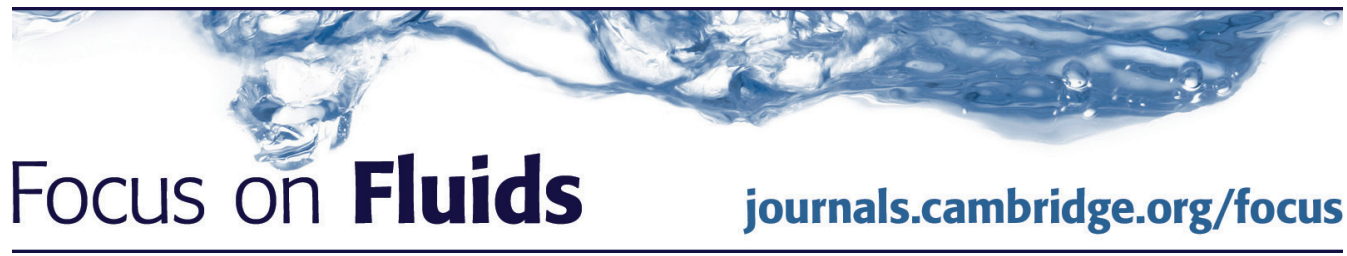

\title{
Solving the inertial particle equation with memory
}

\author{
George Haller $\dagger$ \\ Institute for Mechanical Systems, ETH Zurich, \\ Zurich, Switzerland
}

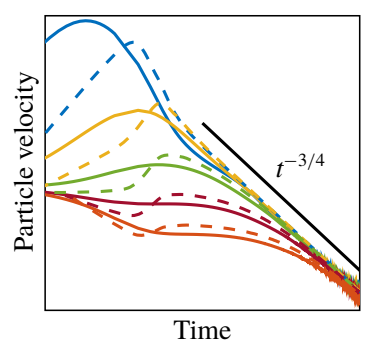

The dynamics of spherical particles in a fluid flow is governed by the well-accepted Maxey-Riley equation. This equation of motion simply represents Newton's second law, equating the rate of change of the linear momentum with all forces acting on the particle. One of these forces, the Basset-Boussinesq memory term, however, is notoriously difficult to handle, which prompts most studies to ignore this term despite ample numerical and experimental evidence of its significance. This practice may well change now due to a clever reformulation of the particle equation of motion by Prasath, Vasan \& Govindarajan (J. Fluid Mech., vol. 868, 2019, pp. 428-460), who convert the Maxey-Riley equation into a one-dimensional heat equation with non-trivial boundary conditions. Remarkably, this reformulation confirms earlier estimates on particle asymptotics, yields previously unknown analytic solutions and leads to an efficient numerical scheme for more complex flow fields.

Key words: computational methods, particle/fluid flow

\section{Introduction}

The motion of finite-size (or inertial) particles immersed in a fluid flow can differ significantly from that of fluid elements. This leads to clustering or scattering effects that cannot be explained using the fluid velocity field alone. The resulting dynamical phenomena are important in a number of areas, including rain drop formation in clouds (Pinsky \& Khain 1997), sinking of biogenic particles in the ocean (Monroy et al. 2017), transport of volcanic ash in the atmosphere (Espinosa Arenal, Avila \& Raza 2018), ocean-drifter motion (Beron-Vera et al. 2015) and cell collection in oncological studies (Haddadi, Naghsh-Nilchi \& Di Carlo 2018), just to name a few.

Most related studies assume that the particles are spherical, do not interact with each other, their associated Stokes and Reynolds numbers are small, the effect of gravity is negligible and the particles do not modify the ambient flow whose velocity field is $\boldsymbol{u}(\boldsymbol{x}, t)$. Under these assumptions, the equation of motion for inertial particles simplifies from its general form derived by Maxey \& Riley (1983) to

$†$ Email address for correspondence: georgehaller@ethz.ch 


$$
\begin{aligned}
R \ddot{\boldsymbol{y}}= & \frac{\mathrm{D} \boldsymbol{u}(\boldsymbol{y}(t), t)}{\mathrm{D} t}-\frac{1}{S}(\dot{\boldsymbol{y}}(t)-\boldsymbol{u}(\boldsymbol{y}(t), t)) \\
& -\sqrt{\frac{3}{S}}\left\{\frac{\dot{\boldsymbol{y}}(0)-\boldsymbol{u}(\boldsymbol{y}(0), 0)}{\sqrt{\pi t}}+\frac{1}{\sqrt{\pi}} \int_{0}^{t} \frac{\dot{\boldsymbol{y}}(s)-\boldsymbol{u}(\boldsymbol{y}(s), s)}{\sqrt{t-s}} \mathrm{~d} s\right\},
\end{aligned}
$$

where $\mathrm{D} / \mathrm{D} t \doteq \partial_{t}+\boldsymbol{u} \cdot \boldsymbol{\nabla}$ is the material derivative along fluid trajectories; $R$ is a dimensionless number depending on the ratio of the particle and fluid densities and $S$ is the Stokes number. The simplified Maxey-Riley (MR) equation given above is a second-order, nonlinear system of implicit integro-differential equations with a singular kernel and with an initial-condition term that blows up as $t \rightarrow 0$. The implicit nature of the equation arises from its last term, the Boussinesq-Basset memory term, which accounts for boundary layer development around the moving particle and renders the equation highly non-trivial to solve. Classical solutions to the MR equation turn out to exist only under the unphysical assumption that the initial particle velocity is exactly equal to the local fluid velocity. In all other cases, only mild solutions can be shown to exist (Farazmand \& Haller 2014; Provencher-Langlois, Farazmand \& Haller 2015). More broadly speaking, the MR equation is not a dynamical system, as the present state does not uniquely determine the future evolution of the inertial particle. Rather, the full history of particle positions and velocities is required for the solution.

Because of these subtleties, the memory term in the MR equation is often omitted in applications (Michaelides 1997). At the same time, growing numerical and experimental evidence for its significance (Candelier, Angilella \& Souhar 2004; Toegel, Luther \& Lohse 2006; Garbin et al. 2009; Daitche \& Tél 2011; Guseva, Feudel \& Tél 2013; Daitche \& Tél 2014) has inspired an ongoing effort to handle this term appropriately in numerical schemes. The main challenge to overcome is the increasing memory cost in many-particle computations, given that the full history of each particle needs to be stored to advance the numerical scheme. To address this challenge, Van Hinsberg, Ten Thije Boonkkamp \& Clercx (2011), Klinkenberg, de Lange \& Brandt (2014), Elghannay \& Tafti (2016) and Parmar et al. (2018) have proposed approximations or modifications to the memory kernel, whereas Daitche (2013) developed higher-order schemes to handle the singularity of the kernel. All these advances, however, involve modifications to the original problem, and assume the initial particle velocity to be equal the flow velocity (i.e. $\dot{y}(0)=\boldsymbol{u}(\boldsymbol{y}(0), 0)$ ), which is unlikely in practical applications.

\section{Overview}

Prasath, Vasan \& Govindarajan (2019) give a fundamentally new take on the MR equation by reformulating (1.1) as a boundary condition for a simple diffusion equation $\boldsymbol{q}_{t}=\boldsymbol{q}_{x x}$ for a vectorial quantity $\boldsymbol{q}(x, t)$, defined over the half-line $x \geqslant 0$ for times $t>0$. Specifically, they select the initial condition $\boldsymbol{q}(x, 0)=\mathbf{0}$ for $x>0$, and the Dirichlet boundary condition $\boldsymbol{q}(0, t)=\boldsymbol{g}_{0}(t)$. They then ask what equivalent Neumann boundary condition would lead to the exact same solution to this problem.

The result is that the equivalent Neumann condition, $\boldsymbol{q}_{x}(0, t)$, for this diffusion problem will be exactly of the form of the bracketed last two terms in the MR equation if one introduces the notation $\boldsymbol{q}(0, t)=\boldsymbol{y}(t)-\boldsymbol{u}(\boldsymbol{y}(t), t)$ in these terms. The remaining terms in (1.1) can be expressed as functions of $\boldsymbol{q}(0, t)$ and $\boldsymbol{q}_{t}(0, t)$, and the $\boldsymbol{y}(t)$ position appearing in the arguments of $\boldsymbol{u}$ and $\mathrm{D} \boldsymbol{u} / \mathrm{D} t$ in the equation can be computed on the run as the solution of the ordinary differential equation $\dot{\boldsymbol{y}}(t)=\boldsymbol{q}(0, t)+\boldsymbol{u}(\boldsymbol{y}(t), t)$. 
This insightful observation enables one to solve the classic diffusion equation for $\boldsymbol{q}(x, t)$, with the MR equation imposed as a Robin-type boundary condition $\boldsymbol{q}_{t}(0, t)+\alpha \boldsymbol{q}(0, t)-\gamma \boldsymbol{q}_{x}(0, t)=\boldsymbol{f}(\boldsymbol{q}(0, t), \boldsymbol{y}, t)$ for appropriate constants $\alpha, \gamma>0$ and a smooth function $\boldsymbol{f}$. In this representation, the original non-local problem, along with all complications of the singular kernel and the integral over the past, disappears. This creates an opportunity to use analytical and numerical methods available for classical diffusion problems to study the dynamics of inertial particles.

As a first illustration of the power of this approach, Prasath et al. (2019) show how particle motion in spatially homogeneous (but still unsteady) flows can be analytically deduced. They derive a formula for $\boldsymbol{q}(0, t)$ which is explicit up to the evaluation of an integral involving the forcing function $\boldsymbol{f}(\boldsymbol{q}(0, t), t)$. In concrete problems, this integral form can be analysed using Watson's lemma (Miller 2006) to obtain asymptotic expressions for the particle velocity for increasing times.

Specifically, the velocity of a particle relaxing in a non-moving background flow $(\boldsymbol{f} \equiv \mathbf{0})$ is found explicitly to decay to zero at a rate of $O\left(t^{-3 / 2}\right)$, confirming the only previously available analytic solution derived for the MR equation by Provencher-Langlois et al. (2015). Notably, ignoring the memory term in the MR equation leads to exponential decay. Sedimenting particles, such as aerosols and droplets in the atmosphere, are characterized by $f \equiv(0$, const. $)$. A similar analysis yields that they approach a vertical terminal velocity at a rate of $O\left(t^{-1 / 2}\right)$, extending the earlier findings of Clift, Grace \& Weber (2013) to the case of non-zero initial velocities. Finally, for a particle in an oscillating background flow $(f \equiv(0, \sin \lambda t))$, the velocity decay to a time-periodic steady state is surprisingly found to be faster in the presence of the Boussinesq-Basset memory term than without it. This shows that the memory term cannot generally be considered as an additional drag on the particle.

For spatially dependent velocity fields, closed-form solutions are generally not available from this approach. An exception is particle migration in planar Couette flow, for which Prasath et al. (2019) still derive a new, explicit solution, exploiting the linearity of the velocity in the spatial variable. This solution may serve as an important benchmark for testing numerical schemes for more general, spatially dependent solutions. In all such schemes, the complete absence of a singular kernel in the diffusive reformulation of the MR equation eliminates any need for approximating such a kernel, keeping the memory requirements low relative to other methods. The numerical scheme proposed by the authors reaches spectral accuracy by employing Chebyshev polynomials in the evaluation of the integrals arising from the nonlinear boundary conditions for the underlying diffusion problem. They illustrate the efficacy of this approach on the example of particle motion in a steady, infinite vortex.

\section{Future}

As many-particle simulations in various media are increasingly popular, the fresh approach of Prasath et al. (2019) will undoubtedly generate further activity in this field. As they have already demonstrated, their reformulation yields new closed-form solutions, provides rigorous asymptotic estimates for the particle velocities and inspires new numerical methods.

Further testing and refinement of the available numerical scheme is highly desirable for problems involving a large number of particles in spatially complex flows with complicated time dependence. In such a challenging setting, a systematic comparison with prior numerical approaches will also be necessary to carry out, factoring in memory requirements, computational speed and coding complexity. Viewing inertial particle motion with memory as a classic diffusion problem may well have presently unforeseen further advantages, as it opens a new connection to a well-developed area of applied mathematics and computational science. 


\section{References}

Beron-Vera, F. J., Olascoaga, M. J., Haller, G., Farazmand, M., Triñanes, J. \& WANG, Y. 2015 Dissipative inertial transport patterns near coherent Lagrangian eddies in the ocean. Chaos 25, 087412.

Candelier, F., Angilella, J. R. \& Souhar, M. 2004 On the effect of the Boussinesq Basset force on the radial migration of a Stokes particle in a vortex. Phys. Fluids 16 (5), 1765-1776.

Clift, R., Grace, J. R. \& Weber, M. E. 2013 Bubbles, Drops, and Particles. Dover.

DAitche, A. 2013 Advection of inertial particles in the presence of the history force: higher order numerical schemes. J. Comput. Phys. 54, 93-106.

DAitche, A.\& TÉL, T. 2011 Memory effects are relevant for chaotic advection of inertial particles. Phys. Rev. Lett. 107, 244501.

Daitche, A. \& TÉL, T. 2014 Memory effects in chaotic advection of inertial particles. New J. Phys. 16 (7), 073008.

Elghannay, H. A. \& TAfTI, D. K. 2016 Development and validation of a reduced order history force model. Intl J. Multiphase Flow 85, 284-297.

Espinosa Arenal, F., Avila, R. \& RaZA, S. S. 2018 A new method of simulating volcanic eruption column formation and dispersion of ejected ash clouds. Meteorol. Appl. 25, 151-160.

Farazmand, M. \& Haller, G. 2014 The Maxey-Riley equation: existence, uniqueness and regularity of solutions. Nonlinear Anal. 22, 98-106.

Garbin, V., Dollet, B., Overvelde, M., Cojoc, D., Di Fabrizio, E., van Wijngaarden, L., Prosperetti, A., de Jong, N., Lohse, D. \& Versluis, M. 2009 History force on coated microbubbles propelled by ultrasound. Phys. Fluids 21 (9), 092003.

Guseva, K., Feudel, U. \& TÉL, T. 2013 Influence of the history force on inertial particle advection: gravitational effects and horizontal diffusion. Phys. Rev. E 88 (4), 042909.

Haddadi, H., Naghsh-Nilchi, H. \& Di CARlo, D. 2018 Separation of cancer cells using vortical microfluidic flows. Biomicrofluidics 12, 014112.

Klinkenberg, J., De LAnge, H. C. \& BrandT, L. 2014 Linear stability of particle laden flows: the influence of added mass, fluid acceleration and Basset history force. Meccanica 49 (4), $811-827$.

MaXey, M. R. \& Riley, J. J. 1983 Equation of motion for a small rigid sphere in a nonuniform flow. Phys. Fluids 26, 883-889.

Michaelides, E. E. 1997 The transient equation of motion for particles, bubbles, and droplets. Trans. ASME J. Fluids Engng 119, 233-247.

Miller, P. D. 2006 Applied Asymptotic Analysis. AMS.

Monroy, P., Hernández-García, E., Rossi, V. \& López, C. 2017 Modeling the dynamical sinking of biogenic particles in oceanic flow. Nonlinear Process. Geophys. 24, 293-305.

Parmar, M., Annamalai, S., Balachandar, S. \& Prosperetti, A. 2018 Differential formulation of the viscous history force on a particle for efficient and accurate computation. J. Fluid Mech. 844, 970-993.

Pinsky, M. \& KhaIn, A. 1997 Turbulence effects on droplets growth and size distribution in clouds a review. J. Aero. Sci. 28, 1177-1214.

Prasath, S. G., Vasan, V. \& Govindarajan, R. 2019 Accurate solution method for the MaxeyRiley equation, and the effects of Basset history. J. Fluid. Mech. 868, 428-460.

Provencher-Langlois, G., Farazmand, M. \& Haller, G. 2015 Asymptotic dynamics of inertial particles with memory. J. Nonlinear Sci. 25, 1225-1255.

Toegel, R., Luther, S. \& Lohse, D. 2006 Viscosity destabilizes sonoluminescing bubbles. Phys. Rev. Lett. 96 (11), 114301.

Van Hinsberg, M. A. T., Ten Thije Boonkkamp, J. H. M. \& Clercx, H. J. 2011 An efficient, second order method for the approximation of the Basset history force. J. Comput. Phys. 230 (4), 1465-1478. 